\title{
Sensorimotor simulations underlie conceptual representations: Modality-specific effects of prior activation
}

\author{
DIANE PECHER \\ Utrecht University, Utrecht, The Netherlands \\ and Erasmus University Rotterdam, Rotterdam, The Netherlands \\ RENÉ ZEELENBERG \\ Indiana University, Bloomington, Indiana \\ and \\ LAWRENCE W. BARSALOU \\ Emory University, Atlanta, Georgia
}

\begin{abstract}
According to the perceptual symbols theory (Barsalou, 1999), sensorimotor simulations underlie the representation of concepts. Simulations are componential in the sense that they vary with the context in which the concept is presented. In the present study, we investigated whether representations are affected by recent experiences with a concept. Concept names (e.g., APPLE) were presented twice in a property verification task with a different property on each occasion. The two properties were either from the same perceptual modality (e.g., green, shiny) or from different modalities (e.g., tart, shiny). All stimuli were words. There was a lag of several intervening trials between the first and second presentation. Verification times and error rates for the second presentation of the concept were higher if the properties were from different modalities than if they were from the same modality.
\end{abstract}

In recent years, researchers have increasingly argued that cognition is based on interactions between sensorimotor systems and the physical world. According to the perceptual symbols theory (Barsalou, 1999), conceptual knowledge is not amodal but instead is grounded in physical experiences. The basic assumption of the theory is that a concept is represented by running a simulation of an actual experience (i.e., of perception and/or action) with that concept. For example, to represent the concept CHAIR, neural systems for vision, action, touch, and emotion partially produce the experience of a particular chair. Behavioral studies increasingly support the view that sensorimotor systems play a critical role in cognition (e.g., Glenberg \& Kaschak, 2002; Pecher, Zeelenberg, \& Barsalou, 2003; Solomon \& Barsalou, 2001; Spivey, Tyler, Richardson, \& Young, 2000; Stanfield \& Zwaan, 2001; Zwaan, Stanfield, \& Yaxley, 2002,Zwaan \& Yaxley, 2003), as does neural evidence (e.g., Martin \& Chao, 2001; Martin, Ungerleider, \& Haxby, 2000; Pulvermüller, 1999).

An important assumption of the perceptual symbols theory (Barsalou, 1999) is that perceptual simulations are componential and dynamic. Rather than act as rigid sym-

Correspondence concerning this article should be sent to D. Pecher, Erasmus University Rotterdam, Department of Psychology, J5-41, Postbus 1738, 3000 DR Rotterdam, The Netherlands (e-mail: pecher@ fsw.eur.nl). bols, perceptual symbols vary widely in their activation patterns, depending on the context in which the concept is encountered (for earlier views on the flexibility of representations, see Anderson et al., 1976; Barsalou, 1982, 1993; Greenspan, 1986; McKoon \& Ratcliff, 1988; Zeelenberg, Pecher, Shiffrin, \& Raaijmakers, 2003). For example, the simulation of APPLE will be different for someone painting a still life than for someone cooking a meal. The simulation will likely include the shape (round) and color (green) of an APPLE for someone who is painting, whereas the simulation will likely include the texture (crunchy) and taste (tart) of an APPLE for someone who is cooking. Thus, simulations may vary in the extent to which information from various modalities is included.

Recently, we have obtained evidence that modalities play an important role in the composition of simulations (Pecher et al., 2003). Using properties from six modalities (vision, audition, taste, smell, touch, and action), we found that there was a modality switch cost in a property verification task in which a different concept was presented on every trial (i.e., concept names were not repeated). For example, subjects might verify the auditory property loud for BLENDER. On the preceding trial, subjects verified a property for a different concept that was either from the same modality (e.g., LEAVES-rustling) or from a different modality (e.g., CRANBERRIES-tart). Responses were faster and more accurate on same-modality trials than on differentmodality trials. This effect could not be explained by as- 
sociative relations between the properties. Rather, the effect is explained by assuming that the simulations are executed by the same systems that are used for perception, and different systems are used for different modalities. For example, to simulate the sound of a BLENDER, the auditory system is used, and to simulate the taste of CRANBERRIES the gustatory system is used. Thus, in the different-modality condition, a cost is associated with switching attention between different perceptual systems, analogous to the modality switching cost observed in perceptual detection studies (e.g., Spence, Nicholls, \& Driver, 2000).

The results of Pecher et al. (2003) are in accordance with the perceptual symbols theory (Barsalou, 1999), which predicts that concepts are represented by sensorimotor systems. It should be noted, however, that amodal theories of conceptual representation might also be able to explain these and other effects that support the perceptual symbols theory. In general, it is very hard or even impossible to design an experiment that provides a critical test of amodal theories, because amodal theories can assume any organization of conceptual knowledge (Barsalou, 1999; Stanfield \& Zwaan, 2001). Hence, an abstract symbols system could account for the results of the Pecher et al. (2003) study if one assumes that conceptual knowledge is organized by modality. This would, however, be a post hoc assumption made to fit the behavioral data, because amodal theories do not make an a priori prediction that conceptual knowledge is organized by modality.

In the present study, we investigated concept-specific long-term consequences of the componentiality of representations. We assumed that if a concept is represented by a simulation that focuses on a specific modality, the next time that the same concept is represented properties from the same modality will be more readily available than properties from a different modality. There is some scarce evidence that prior experiences with a concept may change the availability of specific properties (e.g., Pecher, Zeelenberg, \& Raaijmakers, 1998; Solomon \& Barsalou, 2001; Vriezen, Moscovitch, \& Bellos, 1995), but these studies did not manipulate modality-specific information.

Long-term consequences of modality-specific processing were studied by presenting concept names twice in the experiment. On the first occasion, the concept name was paired with a property name from a specific modalityfor example, APPLE-green (visual) or APPLE-tart (taste). Subjects decided whether the property was true for the concept. According to the perceptual symbols theory, they would perform this task by running a simulation of an APPLE in the relevant modality. After a number of unrelated trials, the concept name was presented again with a different property-for example, APPLE-shiny (visual). If the previous APPLE property was also visual (the same modality condition), running a visual simulation should be easier than if the previous APPLE property was a taste (the different modality condition), because visual properties of APPLE are still more available. Therefore, we expected faster responses and fewer errors in the same-modality condition than in the different-modality condition. The first and second occurrences of a concept were separated by a series of unrelated trials. The length of the lag was varied so that we could consider the time course of recent context effects.

\section{METHOD}

\section{Subjects}

One hundred six volunteers at Utrecht University participated for a monetary fee. All subjects professed to be native speakers of Dutch. The lag between the first and second occurrence of a concept was manipulated between subjects, with $28,28,28$, and 22 subjects in the 12-, 18-, 24-, and 100-trial lag conditions, respectively.

\section{Stimuli}

The critical stimuli consisted of 64 names of concepts, each with 3 names of properties. All concepts were concrete objects. One of the three properties was the target property. The target property could be from one of four modalities: vision, motor action, touch, or sound. For example, vision properties, such as brown or striped, were properties that could be perceived by looking at an object. Motor action properties, such as throw or shake, were actions that could be performed with the object. There were 16 target properties from each of the four modalities. One of the other two properties for a concept was the same-modality property; the other property was the different-modality property. The different modalities included the four target modalities plus taste and smell. For example, for the concept APPLE, the target property was shiny, the same-modality property was green, and the different-modality property was tart. The concept was paired with the target property on its second occurrence. On its first occurrence, the concept was paired either with the same-modality property or with the different-modality property. For each concept, the associative strengths between the same-modality property and the target property and between the different-modality property and the target property were measured in a norming study. The mean associative strengths were smaller than .005 and not reliably different for the same-modality condition and the differentmodality condition. Two counterbalanced lists ensured that across subjects each concept was presented in both conditions.

Different properties were used on the first presentation in the same-modality and the different-modality conditions (note, however, that the target properties were identical). The use of different properties in the two conditions might lead to different amounts of processing (e.g., because one set was more difficult than the other). Therefore, a pilot experiment was done to determine whether there was a difference in difficulty between the two sets of nontarget concept-property pairs (i.e., target properties were not presented in the pilot study). The results showed that there was no reliable difference in performance between the same-modality properties (median $\mathrm{RT}=1,026 \mathrm{msec}$, percent error $=13.4)$ and the differentmodality properties (median $\mathrm{RT}=1,028 \mathrm{msec}$, percent error $=13.7$ ) $[t(55)=0.07$ and $t(55)=0.33$, for RTs and errors, respectively $]$.

In addition to the experimental stimuli, a set of 144 filler stimuli was created. The filler stimuli consisted of a concept name and two property names. The concepts and properties were similar to the experimental ones in that all concepts were objects and all properties were in one of the six modalities. For 64 concepts, the concept was paired with a true property on its first occurrence and with a false property on its second occurrence. For 32 concepts, the concept was paired with a false property on its first occurrence and with a true property on its second occurrence. For the remaining 48 concepts, the concept was paired with a false property on both occurrences. These sets of fillers were smaller than the experimental set to keep the length of the experiment within reasonable limits. For each set, half of the concepts were paired with two properties in the same modality, and the other half were paired with properties in different modalities. 
A third set of 32 stimuli was created for practice. These were similar to the experimental and filler stimuli, with 8 stimuli in each response combination (i.e., true-true, true-false, false-true, falsefalse). Half of each had properties in the same modality, and half had properties in a different modality.

Thus, the total number of stimuli consisted of 240 concepts, presented in 480 trials. The probability of a true trial was slightly higher than that of a false trial, .53 and .47 , respectively. If a concept was paired with a true property on its first occurrence, the probability of a true property on its second occurrence was equal to the probability of a false property on its second occurrence, both .50. If a concept was paired with a false property on its first occurrence, the probability of a true property on its second occurrence was lower than that of a false property on its second occurrence, .42 and .58, respectively. The difference in probabilities between true and false trials (.53 vs. .47) was probably too small to result in a bias to respond "true." If it did, however, it would affect the same- and differentmodality conditions to the same degree. The difference in probabilities between true and false trials after a false property for the same concept may have resulted in a slight bias to respond "false" to concepts for which the response on the first occurrence had also been "false." However, this would have affected only the responses to the filler stimuli. Because the property verification task is quite difficult, we chose to try to keep the number of trials as low as possible rather than have exactly the same number of trials in each response combination.

\section{Procedure}

A trial consisted of the following sequence of events. First, a warning signal $(* * * * *)$ was displayed 10 lines above the center of the computer screen for $500 \mathrm{msec}$. The warning signal was then replaced by the concept name. At the same time, the property name was displayed 4 lines below the concept name. The concept and property names remained on the screen until a response was made. The subjects responded by pressing the "?/" key if they thought that the property was true or by pressing the " $z$ " key if they thought that the property was false for that concept. The subjects were instructed to respond as quickly and as accurately as possible. If the response was incorrect, the message FOUT (error) was displayed for $1,000 \mathrm{msec}$, followed by a 1,000-msec blank screen. If the response was slower than 3,000 msec, the message TE LANGZAAM (too slow) was displayed for $1,000 \mathrm{msec}$, followed by a 500 -msec blank screen. If the response was correct and faster than 3,000 msec, there was no message, and the next trial started $500 \mathrm{msec}$ after the response.

The order of stimuli was randomized with the restriction that the first and second occurrences of a concept were separated by a certain number of trials. The trials were presented in blocks of 80 trials. Between blocks, there was a short break. During the break, the subjects were shown the percentage of errors in the preceding block of trials. If the error percentage was higher than $15 \%$, the subject was instructed to make fewer errors. If the error percentage was lower than $5 \%$, subjects were told that their performance was excellent. The subjects could start the next block of trials by pressing the space bar.

\section{RESULTS}

The means of the subject's median RTs and percent errors on the critical trials are shown in Table 1. RTs for errors and RTs following an error were not included in the calculation of the median RTs. The results indicate that responses were faster and more accurate in the samemodality condition than in the different-modality condition. They also indicate that these effects were more prominent for shorter than for longer lags. Analysis of variance with modality (same vs. different) as a within-subjects factor and lag $(12,18,24,100$ trials) as a between-subjects factor confirmed these observations. RTs were faster for the same-modality condition than for the different-modality condition $\left[F(1,102)=8.95, M S_{\mathrm{e}}=2,679.4, p<.01\right]$. There was no main effect of lag on RTs $(F<1)$, and the interaction between lag and modality condition barely approached significance $\left[F(3,102)=2.09, M S_{\mathrm{e}}=2,679.4\right.$, $p=.106]$. More errors were made in the different-modality condition that in the same-modality condition $[F(1,102)=$ $\left.10.72, M S_{\mathrm{e}}=24.8, p<.01\right]$. There was no main effect of lag on the errors $(F<1)$, and no interaction between lag and modality condition $(F<1)$.

Pairwise comparisons for the RTs showed significant modality effects for the lag 12 and lag 18 conditions $[t(27)=$ $2.58, p<.05$, and $t(27)=2.77, p<.01$, respectively], but no significant effects for the lag 24 and lag 100 conditions $[t(27)=0.64$, and $t(21)=0.14$, respectively $]$. Pairwise comparisons for the error data showed the same pattern with significant modality effects for the lag 12 and lag 18 conditions $[t(27)=2.37, p<.05$, and $t(27)=2.43, p<$ .05 , respectively], but no significant effects for the lag 24 and lag 100 conditions $[t(27)=0.83$, and $t(21)=1.44$, respectively].

\section{DISCUSSION}

We found an effect of sensory modality on performance in a property verification task in which all stimuli were words. These results show that previous experiences with a concept influence later activation of the same concept. Property verification was faster and more accurate when the concept was previously presented with a property from the same modality as that for the current property than when it was presented with a property from a different modality. Although the interaction between modality and lag was not significant, the modality effect seemed to be

Table 1

Mean Median Reaction Times (RTs, in Milliseconds) and Percent Errors (PEs) as a Function of Modality Condition and Lag (Number of Unrelated Trials)

\begin{tabular}{|c|c|c|c|c|c|c|c|c|}
\hline \multirow[b]{3}{*}{ Condition } & \multicolumn{8}{|c|}{ Lag } \\
\hline & \multicolumn{2}{|c|}{12} & \multicolumn{2}{|c|}{18} & \multicolumn{2}{|c|}{24} & \multicolumn{2}{|c|}{100} \\
\hline & $\mathrm{RT}$ & $\mathrm{PE}$ & $\mathrm{RT}$ & $\mathrm{PE}$ & RT & $\mathrm{PE}$ & RT & $\mathrm{PE}$ \\
\hline Same modality & 992 & 13.5 & 983 & 12.8 & 978 & 12.7 & 1,005 & 12.8 \\
\hline Different modality & 1,026 & 16.5 & 1,024 & 15.9 & 985 & 14.2 & 1,002 & 14.1 \\
\hline Same-modality advantage & 34 & 3.0 & 41 & 3.1 & 7 & 1.5 & -3 & 1.3 \\
\hline
\end{tabular}


more pronounced for shorter lags than for longer lags. Pairwise comparisons showed significant modality effects in both RT and error rates for the shorter but not for the longer lags.

The present results provide evidence for the assumption that representations are componential and not holistic. If simulations were holistic, all modalities should be involved, and thus there should be no difference between the samemodality and the different-modality conditions. The results from the present study offer a good indication of the mechanism behind the recency effect. On the first occurrence of the concept, a simulation is run in the modality that is relevant for verifying the property. The simulation may result in a temporary increase in the availability of properties in that modality. If on a second occurrence of the same concept this modality is again relevant for verifying a property, performance is expected to be better than if the first modality is irrelevant. Thus, the modalities involved in simulations of concepts are not only affected by the current context but also by recent experiences with that concept. ${ }^{1}$

There is a growing body of evidence for the role of sensorimotor systems in cognition (Glenberg \& Kaschak, 2002; Solomon \& Barsalou, 2001; Spivey et al., 2000; Stanfield \& Zwaan, 2001; Zwaan et al., 2002; Zwaan \& Yaxley, 2003). All these studies have shown that variables that affect perception and action also affect cognitive processes. A strong assumption of the perceptual systems theory is that the same neural states that underlie perception and action also underlie conceptual representations. There is some evidence from neuroimaging and ERP studies showing that sensorimotor areas are activated during semantic word processing tasks (e.g., Martin \& Chao, 2001; Martin et al., 2000; Pulvermüller, 1999). Thus, concepts may be represented by the same neural systems as those with which they are perceived.

\section{REFERENCES}

Anderson, R. C., Pichert, J. W., Goetz, E. T., Schallert, D. L., STEVEns, K. V., \& TROLlip, S. R. (1976). Instantiation of general terms. Journal of Verbal Learning \& Verbal Behavior, 15, 667-679.

BARSALOU, L. W. (1982). Context-independent and context-dependent information in concepts. Memory \& Cognition, 10, 82-93.

BARSALOU, L. W. (1993). Flexibility, structure, and linguistic vagary in concepts: Manifestations of a compositional system of perceptual symbols. In A. F. Collins, S. E. Gathercole, M. A. Conway, \& P. E. Morris (Eds.), Theories of memory (pp. 30-89). Hove, U.K.: Erlbaum.

Barsalou, L. W. (1999). Perceptual symbol systems. Behavioral \& Brain Sciences, 22, 577-660.

GlenberG, A. M., \& KASCHAK, M. P. (2002). Grounding language in action. Psychonomic Bulletin \& Review, 9, 558-565.

Greenspan, S. L. (1986). Semantic flexibility and referential specificity of concrete nouns. Journal of Memory \& Language, 25, 539-557.
Martin, A., \& CHAO, L. L. (2001). Semantic memory and the brain: Structure and processes. Current Opinion in Neurobiology, 11, 194201.

Martin, A., Ungerleider, L. G., \& Haxby, J. V. (2000). Categoryspecificity and the brain: The sensory-motor model of semantic representations of objects. In M. S. Gazzaniga (Ed.), The new cognitive neurosciences (2nd ed., pp. 1023-1036). Cambridge, MA: MIT Press.

McKoon, G., \& RATCLIFF, R. (1988). Contextually relevant aspects of meaning. Journal of Experimental Psychology: Learning, Memory, \& Cognition, 14, 331-343.

Pecher, D., Zeelenberg, R., \& Barsalou, L. W. (2003). Verifying different-modality properties for concepts produces switching costs. Psychological Science, 14, 119-124.

Pecher, D., Zeelenberg, R., \& RaAijmakers, J. G. W. (1998). Does pizza prime coin? Perceptual priming in lexical decision and naming. Journal of Memory \& Language, 38, 401-418.

Pulvermüller, F. (1999). Words in the brain's language. Behavioral \& Brain Sciences, 22, 253-336.

SOLOMON, K. O., \& BARSALOU, L. W. (2001). Representing properties locally. Cognitive Psychology, 43, 129-169.

Spence, C., Nicholls, M. E. R., \& Driver, J. (2000). The cost of expecting events in the wrong sensory modality. Perception \& Psychophysics, 63, 330-336.

Spivey, M., Tyler, M., Richardson, D., \& Young, E. (2000). Eye movements during comprehension of spoken scene descriptions. In Proceedings of the 22nd Annual Conference of the Cognitive Science Society (pp. 487-492). Mahwah, NJ: Erlbaum.

STANFIELD, R. A., \& ZWAAN, R. A. (2001). The effect of implied orientation derived from verbal context on picture recognition. Psychological Science, 12, 153-156.

Vriezen, E. R., Moscovitch, M., \& Bellos, S. A. (1995). Priming effects in semantic classification tasks. Journal of Experimental Psychology: Learning, Memory, \& Cognition, 21, 933-946.

Zeelenberg, R., Pecher, D., Shiffrin, R. M., \& RaAijmakers, J. G. W. (2003). Semantic context effects and priming in word association. Psychonomic Bulletin \& Review, 10, 653-660.

ZwaAn, R. A., StAnfield, R. A., \& YAXley, R. H. (2002). Language comprehenders mentally represent the shape of objects. Psychological Science, 13, 168-171.

ZWAAN, R. A., \& YAXLEY, R. H. (2003). Spatial iconicity affects semantic relatedness judgments. Psychonomic Bulletin \& Review, 10, 954-958.

\section{NOTE}

1. Note that the nature of the described mechanism is facilitatory. The somewhat longer RTs in the different-modality condition in the shorter lag conditions, however, might seem to support an inhibitory account of the modality effect. It should be noted, though, that lag was manipulated between subjects. Hence, it could simply be that overall, subjects in the shorter lag conditions were somewhat slower than subjects in the longer lag conditions. An analysis of the practice trials data was consistent with this interpretation: RTs for the practice trials also showed slower responses for the shorter lag conditions. Thus, it is very likely that on the average the subjects in the shorter lag conditions simply happened to be slightly slower than those in the longer conditions.

(Manuscript received October 29, 2002; revision accepted for publication February 5, 2003.) 